\title{
Reconstruction of the elbow using pedicle joint freezing after wide excision for soft tissue sarcoma: A case report
}

\author{
YUTA HASHIMOTO $^{1}$, YASUNORI TOME ${ }^{1}$, HIROMICHI OSHIRO ${ }^{1}$, \\ YUSUKE AOKI $^{1}$, HIROKI MAEHARA ${ }^{2}$ and KOTARO NISHIDA ${ }^{1}$ \\ ${ }^{1}$ Department of Orthopedic Surgery, Graduate School of Medicine, University of the Ryukyus; \\ ${ }^{2}$ Department of Hyperbaric Medicine, University of the Ryukyus Hospital, Nishihara, Okinawa 903-0125, Japan
}

Received November 2, 2020; Accepted March 19, 2021

DOI: $10.3892 / \mathrm{mco} .2021 .2277$

\begin{abstract}
A 35-year-old man presented with a four-year history of a growing mass on the anterior aspect of his left elbow. Magnetic resonance imaging revealed a soft tissue tumor in the brachialis muscle extending to the cubital fossa. Following an open biopsy, the tumor was diagnosed as a monophasic fibrous synovial sarcoma. After neoadjuvant chemotherapy, the patient underwent wide excision and reconstruction of the elbow joint with a pedicle frozen autograft. At the final follow-up four years after surgery, the elbow range of motion was $0-120^{\circ}$. Although there were signs of osteoarthritis, there was no narrowing of the joint -, and the patient experienced only mild pain. To the best of our knowledge, the present case report is the first to describe wide tumor excision and reconstruction using a pedicle frozen autograft of the elbow. This method should be considered after excision of malignant bone and soft tissue tumors, especially in non-weight-bearing joints. Further cases have to be evaluated to understand the complications and long-term prognosis of this procedure.
\end{abstract}

\section{Introduction}

Synovial sarcoma, which accounts for $5-10 \%$ of all soft tissue sarcomas, has a high prevalence among males $(1,2)$. Synovial sarcoma is the most frequent soft tissue sarcoma in children and young adults, but its peak incidence occurs in the third decade of life $(3,4)$. The most frequently affected sites are the extremities. However, synovial sarcoma can develop from serosal surfaces, such as the pleura and pericardium $(4,5)$. Synovial sarcoma has two pathological subtypes: Monophasic and biphasic. Moreover, synovial sarcoma has unique genomic characteristics, such as the translocation $\mathrm{t}(\mathrm{X} ; 18)(\mathrm{p} 12.2: \mathrm{q} 11: 2)(6)$. The standard

Correspondence to: Dr Yasunori Tome, Department of Orthopedic Surgery, Graduate School of Medicine, University of The Ryukyus, 207 Uehara, Nishihara, Okinawa 903-0125, Japan

E-mail: yastome@med.u-ryukyu.ac.jp

Key words: case report, elbow, frozen autograft, pedicle freezing, reconstructive surgical procedure, synovial sarcoma treatment for localized synovial sarcoma is surgical resection with adequate margins (7).

Several oncological reconstructions, such as megaprostheses or biological reconstructions using irradiation, autoclaving, pasteurization, or freezing, have been developed for the bone defects after wide excision of malignant bone or soft tissue sarcoma. Tsuchiya et al (8) developed a frozen autograft technique. In the early days of the technique, the affected bone was excised with an appropriate surgical margin. The resected bones were frozen and consecutively reconstructed with intramedullary nails or plates. Later, Shimozaki et al (9) modified this freezing method and developed a pedicle freezing method. The pedicle freezing method allows maintenance of the anatomical continuity on either the proximal or distal side. This modified pedicle freezing method may potentially achieve a higher bone union rate and limb function than those achieved with the original free-freezing method.

In this case report, we discuss the excision of a synovial sarcoma close to the elbow and the successful reconstruction with a pedicle frozen autograft involving the elbow joint. To our knowledge, this is the first reconstruction that used a pedicle frozen autograft involving the joint after the wide excision of a soft tissue tumor in the elbow.

\section{Case report}

Case history. A 35-year-old man presented to our institution with a four-year history of a growing mass on the anterior surface of his left elbow. Magnetic resonance imaging revealed a soft tissue tumor in the brachialis muscle that extended into the cubital fossa and measured $13.6 \times 4.7 \times 4.0 \mathrm{~cm}$ in size (Fig. 1A and B). We found no distant metastases on ${ }^{18} \mathrm{~F}$-fluorodeoxyglucose positron emission tomography. Following an open biopsy, histopathological examination allowed the diagnosis of a monophasic fibrous synovial sarcoma. As stipulated by our institution's standard chemotherapeutic regimen, the patient received three courses of neoadjuvant chemotherapy with intravenous doxorubicin and ifosfamide.

Surgical technique (therapeutic intervention). After neoadjuvant chemotherapy, the patient underwent wide excision and reconstruction of his elbow joint. First, two longitudinal skin 
incisions were made on the anteromedial and posterior aspects of the elbow. Then, we excised the tumor together with the brachialis and biceps muscles through the anteromedial incision (Fig. 2A). We performed careful in situ preparation (10) to preserve the musculocutaneous and median nerves and brachial vessels close to the surgical margin. We performed a wide excision without sacrificing these major vessels and nerves. In the next step, we isolated the tumor, including its soft tissue margin, using the vinyl sheet but ensuring the continuity of the vessels and nerves (Fig. 2A). Within the vinyl sheet, we carefully dissected the vessels and nerves from the soft tissue before the tumor was resected (Fig. 2B). The preserved vessels and nerves were washed with $100 \%$ ethanol and distilled water to reduce the risk of local recurrence (10). In the next step, we dislocated the radial head so that we could isolate the humeroulnar joint. We performed a z-shaped osteotomy of the ulna at approximately $8 \mathrm{~cm}$ from its proximal end. Finally, the humeroulnar joint, the proximal part of the ulna, and the distal humerus were isolated, the latter at $13 \mathrm{~cm}$ proximal to its distal end, in preparation for pedicle freezing. After the tumor-bearing part of the humerus was sufficiently isolated, the tumor and surrounding soft tissues were removed (Fig. 2C). Pedicle freezing was performed under tourniquet control to avoid or at least reduce tumor dissemination. The humerus, humeroulnar joint, and proximal ulna were immersed in liquid nitrogen and frozen for $20 \mathrm{~min}$ (Fig. 2C). After thawing at room temperature for $15 \mathrm{~min}$ (Fig. 2D and E), we washed the autograft with a solution of distilled water and $1 \%$ iodine for $15 \mathrm{~min}$ to reduce the risk of infection. The proximal ulna was fixed with a locking compression plate, and a suture anchor was used to reattach the radial collateral ligament.

Postoperative course (follow-up and outcomes). The elbow was immobilized with a splint for three weeks. Thereafter, elbow mobilization was initiated, and two courses of adjuvant chemotherapy with intravenous doxorubicin and ifosfamide were administered. Seven months postoperatively, radiography demonstrated complete bone union at the ulnar osteotomy site (Fig. 3A). The elbow's range of motion was normal with $0^{\circ}$ of extension, $120^{\circ}$ of flexion, $70^{\circ}$ of pronation, and $90^{\circ}$ of supination. Three years after surgery, lung metastases were found and resected using video-assisted thoracic surgery. Four years after the autograft reconstruction, osteophytes and osteosclerosis, but no signs of humeroulnar joint narrowing were visible on radiography (Fig. 3B). The International Society of Limb Salvage (ISOLS) score was $83 \%$ at the final follow-up four years postoperatively with neither recurrence nor further metastases. The patient was able to work as an electronic engineer and play bass guitar.

\section{Discussion}

The standard therapeutic approach for synovial sarcoma is wide surgical excision combined with neoadjuvant and adjuvant chemotherapy and/or radiotherapy $(1,5,7,11)$. The frozen autograft technique of Tsuchiya et al (8) uses liquid nitrogen at $-196^{\circ} \mathrm{C}$ as the cryogenic agent to destroy tumor cells. Freezing at this extremely low temperature devitalizes tumor cells by inducing ice crystal formation and cell dehydration (12). In our case, we used the pedicle freezing method for elbow reconstruction after wide excision of a synovial sarcoma for the first time. Complete bone union of the ulnar z-graft was achieved by the seventh month after surgery, and upper extremity function was fully preserved. Although radiological signs of osteoarthritis of the elbow joint were found at the follow-up after four years, there was no joint space narrowing, and the patient was not affected in his activities of daily living.

The advantages of using frozen autografts are as follows: i) They are easy to perform; ii) They require only a short duration of treatment; iii) They fit perfectly because the anatomy remains unchanged; iv) There is no need for bone banking; v) They are easy to attach to any preserved tendons and ligaments; and vi) They achieve earlier osteogenesis do than pasteurized autografts $(13,14)$. Zekry et al found that the fiveand ten-year survival rates of frozen autografts were as high as $91.2 \%$, and that they achieved complete bony union in $97 \%$ of patients after sarcoma or bone metastasis excision (15). Based on these findings, we chose to perform a frozen autograft in our patient to ensure bone and joint preservation.

Most descriptions of the use of frozen autografts for reconstruction after wide excision of sarcomas involve the lower extremities. There are some reports of biological reconstruction around the elbow involving free frozen autografts and flaps (16). Compared to a free frozen autograft, pedicle frozen autografts have shown a shorter bone union period and lower postoperative complication rates (9). In our case, because the sarcoma was adjacent to both the humerus and ulna, we immersed the humerus and ulna with the elbow joint in liquid nitrogen during the pedicle joint freezing to preserve the limb function. Bone union at the osteotomy site was achieved seven months after surgery, and the patient showed full elbow joint range of motion.

There are different methods described that preserve elbow function. In patients with implant use for prosthetic replacement following the resection of malignant or progressive benign tumors around the elbow, the survival rate was $64 \%$ after five years (17). However, the humeral stem loosening rate was high (17). There are several bone recycling methods, such as autoclaving, irradiation, and pasteurization. However, these techniques may cause heat- or radiation-induced bone weakness (18). Some studies involving histological examinations of these autografts found that osteogenesis occurred only slowly $(13,14)$.

Postoperative osteoarthritis is a long-term complication of frozen autografts. A study on frozen autografts after resection of malignant bone tumors (mostly osteosarcomas) with epiphyseal involvement compared 27 patients with a mean age of 31.6 years (range 12-72 years) and a mean follow-up period of 94.0 months (range 6-217 months). They found that only 1 of 23 patients in the total epiphyseal freezing group remained osteoarticular graft survived until the final follow-up (19). The other 22 patients had developed osteoarthritic changes by the final follow-up (19). Meanwhile, all four of the patients who underwent intraepiphyseal resection for partial preservation of the healthy cartilage had excellent osteoarticular graft survival (19). However, most of the cases in their report involved lower extremity joints, and no elbow joints were described. In our patient, we observed no narrowing of the humeroulnar joint four years after the surgery. As the elbow joint is not a weight-bearing joint, osteoarthritis may progress more slowly than what has been observed in lower extremity joints. 

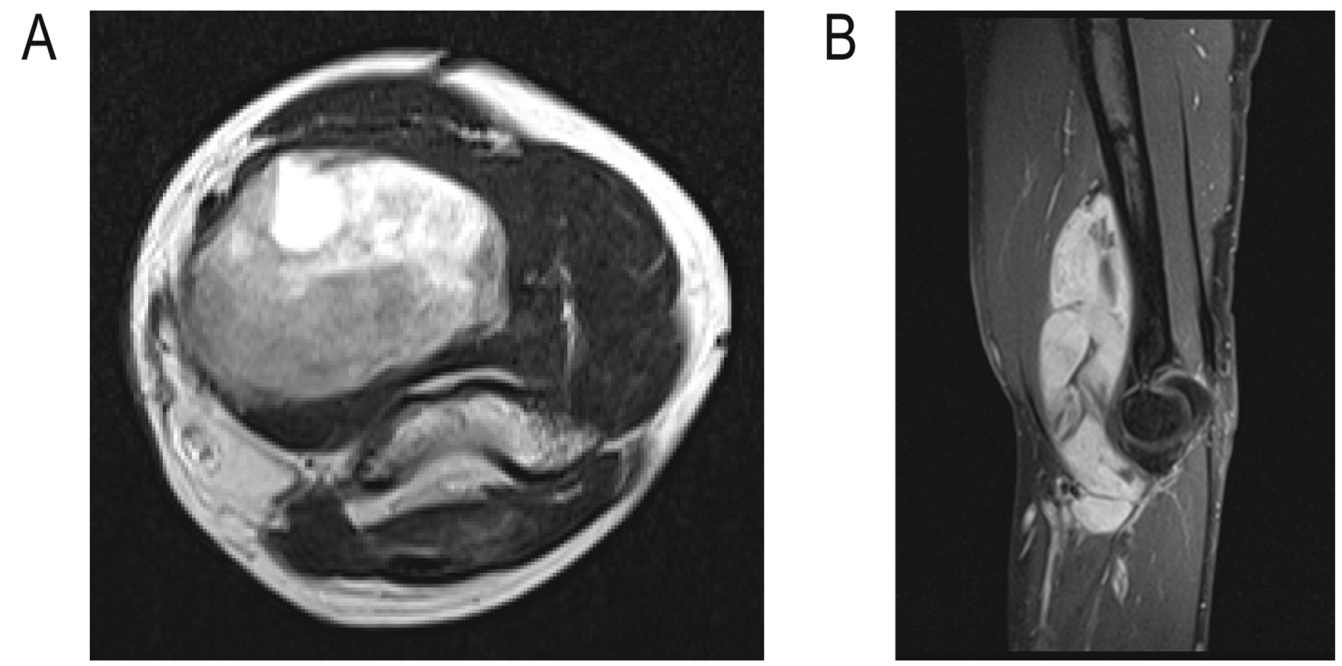

Figure 1. A 35-year-old man with a monophasic fibrous synovial sarcoma in his left elbow. Magnetic resonance imaging located the tumor in the brachialis muscle of the forearm. It had a high signal intensity on T2-weighted and gadolinium-enhanced T1-weighted imaging. (A) Axial view of the T2-weighted imaging. (B) Sagittal view of the gadolinium-enhanced fat-suppressed T1-weighted imaging.
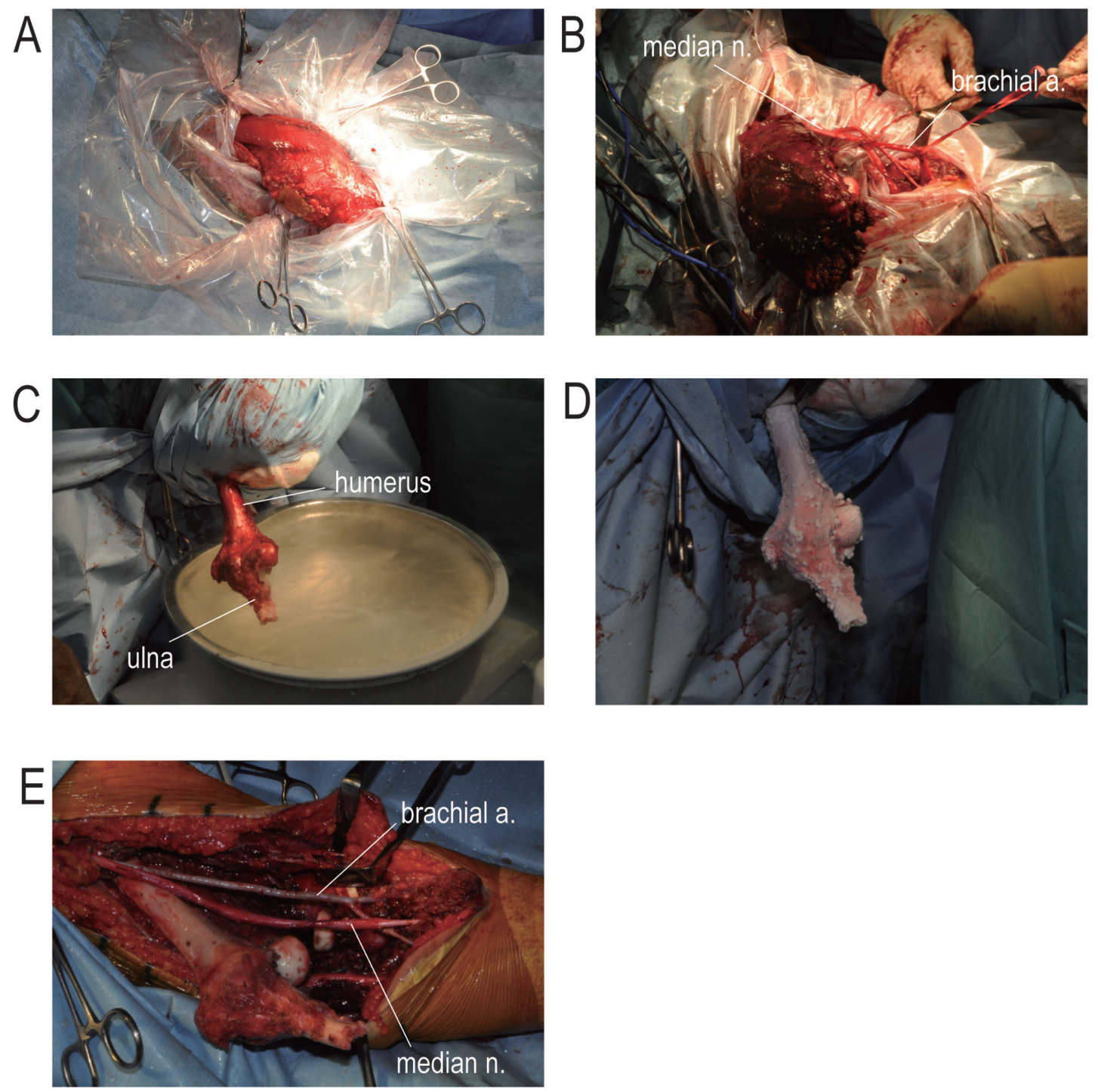

Figure 2. Intraoperative images in a 35-year-old man with a monophasic fibrous synovial sarcoma in his left elbow. (A) Wide excision of the tumor and separation of the proximal radioulnar joint was performed anteromedially of the elbow. (B) In situ preparation: The musculocutaneous and median nerves, and the brachial, radial and ulnar arteries were carefully dissected within the vinyl sheet. (C) Humerus, humeroulnar joint and proximal ulna were immersed in liquid nitrogen and frozen for $20 \mathrm{~min}$. (D) Autograft was thawed at room temperature for $15 \mathrm{~min}$. (E) Autograft was returned to its original position. a, artery; n, nerve. 

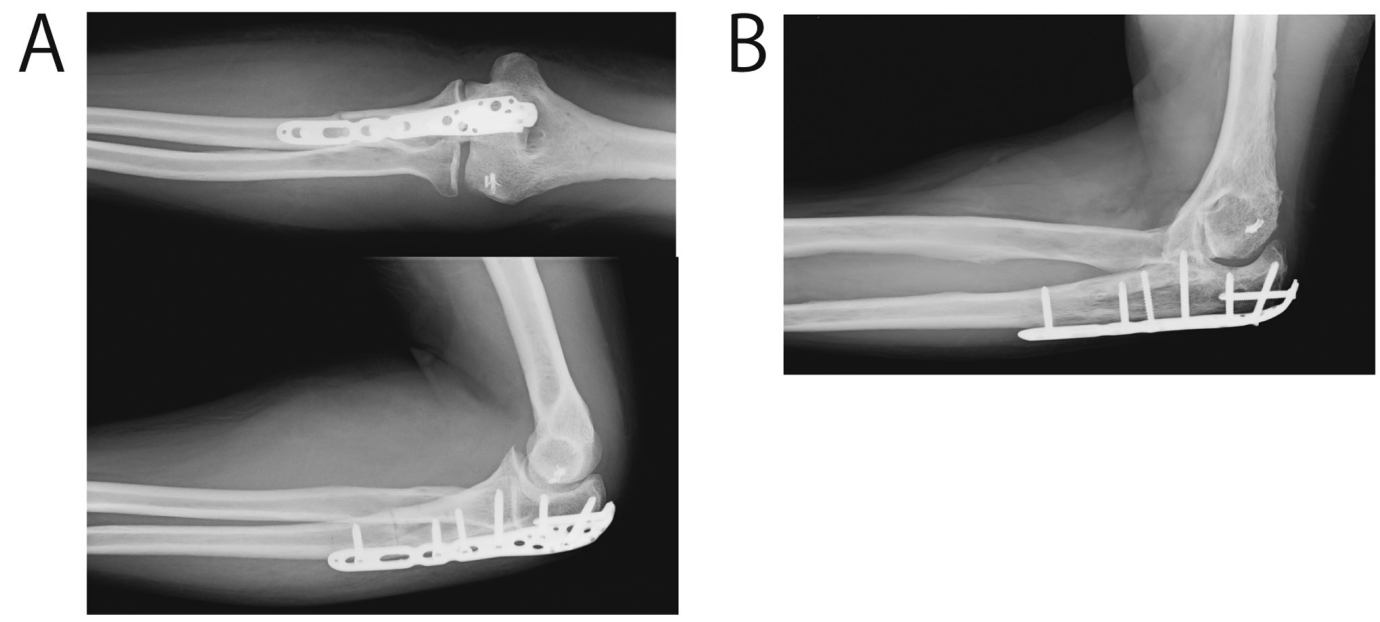

Figure 3. Postoperative X-ray images after resection of a synovial sarcoma of the left elbow in a 35-year-old man. (A) Front (upper panel) and lateral (bottom panel) views seven months after surgery: Bone union was achieved at the ulnar osteotomy site, and there were no fractures. (B) Lateral view four years after surgery: Osteophytes and osteosclerosis can be seen, but no signs of humeroulnar joint narrowing.

We describe the first case of wide excision and reconstruction with pedicle freezing of the elbow joint in a patient with a monophasic fibrous synovial sarcoma. Based on our experience with this case, we consider this a promising method for reconstruction after the excision of malignant bone or soft tissue tumors around the elbow joint. More patients need to be treated and followed up to assess the complications and long-term prognosis of this method.

In conclusion, to the best of our knowledge, this is the first case of a pedicle frozen autograft involving the elbow joint following the wide excision of a soft tissue tumor. Although the reconstruction is challenging due to the complex anatomy of the elbow, pedicle joint freezing combined with in situ preparation may help to preserve the limb and the function of the elbow joint.

\section{Acknowledgements}

Not applicable.

\section{Funding}

No funding was received.

\section{Availability of data and materials}

The datasets used and/or analyzed during the current study are available from the corresponding author on reasonable request.

\section{Authors' contributions}

YH, YT, HO, YA, HM and KN wrote and edited the manuscript. YT and HM performed the surgery and postoperative management. YT critically revised the manuscript. YH and YT are responsible for confirming the authenticity of the raw data. All authors read and approved the final manuscript.

\section{Ethics approval and consent to participate}

Ethics approval is not applicable since this case report was considered part of routine follow-up treatment of the patient.
Written informed consent was obtained from the patient in this case report.

\section{Patient consent for publication}

Written consent for publication was obtained from the patient in the present case report.

\section{Competing interests}

The authors declare that they have no competing interests.

\section{References}

1. Kottu R and Prayaga AK: Synovial sarcoma with relevant immunocytochemistry and special emphasis on the monophasic fibrous variant. J Cytol 27: 47-50, 2010.

2. Riedel RF, Jones RL, Italiano A, Bohac C, Thompson JC, Mueller K, Khan Z, Pollack SM and van Tine BA: Systemic anti-cancer therapy in synovial sarcoma: A systematic review. Cancers (Basel) 10: 417, 2018.

3. Ferrari A, De Salvo GL, Brennan B, van Noesel MM, De Paoli A, Casanova M, Francotte N, Kelsey A, Alaggio R, Oberlin O, et al: Synovial sarcoma in children and adolescents: The European pediatric soft tissue sarcoma study group prospective trial (EpSSG NRSTS 2005). Ann Oncol 26: 567-572, 2015.

4. Kerouanton A, Jimenez I, Cellier C, Laurence V, Helfre S, Pannier S, Mary P, Freneaux P and Orbach D: Synovial sarcoma in children and adolescents. J Pediatr Hematol Oncol 36: 257-262, 2014.

5. Wang S, Song R, Sun T, Hou B, Hong G, Mallampati S, Sun H, Zhou X, Zhou C, Zhang H, et al: Survival changes in patients with synovial sarcoma, 1983-2012. J Cancer 8: 1759-1768, 2017.

6. Ladanyi M: Fusions of the SYT and SSX genes in synovial sarcoma. Oncogene 20: 5755-5762, 2001.

7. Singer S, Baldini EH, Demetri GD, Fletcher JA and Corson JM: Synovial sarcoma: Prognostic significance of tumor size, margin of resection, and mitotic activity for survival. J Clin Oncol 14: 1201-1208, 1996.

8. Tsuchiya H, Wan SL, Sakayama K, Yamamoto N, Nishida H and Tomita K: Reconstruction using an autograft containing tumour treated by liquid nitrogen. J Bone Joint Surg Br 87: 218-225, 2005.

9. Shimozaki S, Yamamoto N, Shirai T, Nishida H, Hayashi K, Tanzawa Y, Kimura H, Takeuchi A, Igarashi K, Inatani $\mathrm{H}$, et al: Pedicle versus free frozen autograft for reconstruction in malignant bone and soft tissue tumors of the lower extremities. J Orthop Sci 19: 156-163, 2014. 
10. Matsumoto S, Kawaguchi N, Manabe J and Matsushita $\mathrm{Y}$ : 'In situ preparation': New surgical procedure indicated for soft-tissue sarcoma of a lower limb in close proximity to major neurovascular structures. Int J Clin Oncol 7: 51-56, 2002.

11. Pasquali S and Gronchi A: Neoadjuvant chemotherapy in soft tissue sarcomas: Latest evidence and clinical implications. Ther Adv Med Oncol 9: 415-429, 2017.

12. Yamamoto $\mathrm{N}$, Tsuchiya $\mathrm{H}$ and Tomita $\mathrm{K}$ : Effects of liquid nitrogen treatment on the proliferation of osteosarcoma and the biomechanical properties of normal bone. J Orthop Sci 8: 374-380, 2003.

13. Tanzawa Y, Tsuchiya H, Shirai T, Hayashi K, Yo Z and Tomita K Histological examination of frozen autograft treated by liquid nitrogen removed after implantation. J Orthop Sci 14: 761-768, 2009.

14. Ehara S, Nishida J, Shiraishi H and Tanakawa Y: Pasteurized intercalary autogeneous bongraft: Radiographic and scintigraphic features. Skeletal Radiol 29: 335-339, 2000.

15. Zekry KM, Yamamoto N, Hayashi K, Takeuchi A, Higuchi T, Abe K, Taniguchi Y, Alkhooly AZ, Abd-Elfattah AS, Fouly EH, et al: Intercalary frozen autograft for reconstruction of malignant bone and soft tissue tumours. Int Orthop 41: 1481-1487, 2017.

16. Hirakawa A, Nagano A, Komura S, Ishimaru D, Kawashima K and Akiyama $\mathrm{H}$ : Reconstruction of a malignant soft tissue tumor around the elbow joint using a frozen autograft treated with liquid nitrogen, in combination with a free anterolateral thigh flap: A report of two cases. Case Reports Plast Surg Hand Surg 5: $80-86,2018$
17. Henrichs MP, Liem D, Gosheger G, Streitbuerger A, Nottrott M, Andreou D and Hardes J: Megaprosthetic replacement of the distal humerus: Still a challenge in limb salvage. J Shoulder Elbow Surg 28: 908-914, 2019.

18. Singh VA, Nagalingam J, Saad M and Pailoor J: Which is the best method of sterilization of tumour bone for reimplantation? A biomechanical and histopathological study. Biomed Eng Online 9: 48, 2010.

19. Hayashi K, Yamamoto N, Takeuchi A, Miwa S, Igarashi K, Higuchi T, Abe K, Taniguchi Y, Aiba H, Araki Y, et al: Clinical course of grafted cartilage in osteoarticular frozen autografts for reconstruction after resection of malignant bone and soft-tissue tumor involving an epiphysis. J Bone Oncol 24: 100310, 2020.

This work is licensed under a Creative Commons Attribution-NonCommercial-NoDerivatives 4.0 International (CC BY-NC-ND 4.0) License. 